Palavras chave: Calophyllum brasiliense Cambèss Nitrogênio Adubação

Histórico: Recebido 18/01/2012 Aceito 29/04/2014

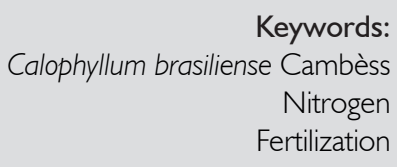

Correspondência: valeriaciriello@tropicalflora.com.br

DOI: | 0. I590/0 I04776020|42004|445

\section{DOSES DE NITROGÊNIO NO CRESCIMENTO INICIAL E NUTRIÇÃO DE PLANTAS DE GUANANDI}

RESUMO: Conduziu-se, este trabalho, com o objetivo de avaliar o efeito de doses de nitrogênio sobre o desenvolvimento inicial e nutrição de plantas de Guanandi (Calophyllum brasiliense Cambèss), espécie arbórea nativa brasileira. $O$ experimento foi realizado em casa de vegetação, em recipientes plásticos com capacidade de $50 \mathrm{~L}$ de solo tipo Latossolo vermelho distrófico. $O$ delineamento experimental utilizado foi inteiramente casualizado, com seis tratamentos e quatro repetições. Os tratamentos constituíram-se de cinco doses de N: 0, 40, 80, 120 e $160 \mathrm{mg} \mathrm{dm}^{-3}$, mais o controle (sem adubação e calagem), sendo a fonte de adubação utilizada a ureia. Com exceção do controle, todos os tratamentos receberam calagem para elevar a saturação por bases do solo de $60 \%$ e adubação com $150 \mathrm{mg} \mathrm{dm}^{-3}$ de $\mathrm{P}_{2} \mathrm{O}_{5}, 80 \mathrm{mg} \mathrm{dm}^{-3}$ de $\mathrm{K}_{2} \mathrm{O}$. Foram analisados mensalmente a altura da planta e diâmetro do coleto e aos 10 meses de idade: a massa seca de folhas, caules, raízes e total, a área foliar e a análise química das folhas e caules. As doses de $\mathrm{N}$ afetaram, significativamente, os dados de altura, área foliar, massa seca do caule e massa seca foliar, observando-se um efeito quadrático; já, os dados de diâmetro e massa seca de raízes foram afetados de forma linear negativa. A melhor resposta de crescimento foi obtida com a dose de $40 \mathrm{mg} \mathrm{dm}^{-3}$ de $\mathrm{N}$.

\section{NITROGEN DOSES ON THE INITIAL GROWTH AND NUTRITION OF GUANANDI PLANTS}

ABSTRACT: The objective of this study was to evaluate the effect of nitrogen doses on the initial development and nutrition of Guanandi (Calophyllum brasiliense Cambèss) plants, a native Brazilian arboreal species. The experiment was carried out in a greenhouse, in plastic containers with capacity of $50 \mathrm{~L}$ of dystrophic red Latosol (oxisol). The experimental design used was completely randomized, with six treatments and four repetitions. The treatments consisted of five $\mathrm{N}$ doses: $0,40,80,120$ and $160 \mathrm{mg} \mathrm{dm}^{-3}$, and the control (with no fertilization or liming of the soil), where urea was used as the fertilization source. With the exception of the control, all the treatments received liming to elevate saturation by bases of soil of $60 \%$ and fertilization with $150 \mathrm{mg} \mathrm{dm}^{-3}$ of $\mathrm{P}_{2} \mathrm{O}_{5}, 80 \mathrm{mg} \mathrm{dm}$ of $\mathrm{K}_{2} \mathrm{O}$. There were monthly analysis of the plant's height and diameter of the connecting point of root and stem of the plant and at its 10 months age were also analyzed the leaves, stems, roots and total dry mass, the foliar area and the chemical analysis of leaves and stems. The $\mathrm{N}$ doses significantly affected the height, foliar area, stem and foliar dry mass data, observing a quadratic effect. The diameter and root dry mass data were affected on a negative linear way. The best growth response was obtained with the $40 \mathrm{mg} \mathrm{dm}^{-3} \mathrm{~N}$ dose.

I Tropical Flora Reflorestadora - Garça, São Paulo, Brasil

2 Universidade Estadual Paulista Julio de Mesquita Filho - Botucatu, São Paulo, Brasil

${ }^{3}$ Universidade Estadual de Goiás - São Luís de Montes Belos, Goiás, Brasil 


\section{INTRODUÇÃO}

A demanda por madeira no Brasil vem aumentando a cada ano e com ela a pressão contra o desmatamento das florestas nativas. Esse fato faz com que aumente a necessidade de reflorestamento comercial de espécies madeireiras.

Dentre as espécies nativas que vêm sendo utilizadas para fins comerciais destaca-se o guanandi, uma espécie com ampla distribuição tropical que tem sido plantada comercialmente em diversos países da América Latina. Sua importância tem crescido pelo fato de sua madeira ser resistente à água e ter boa durabilidade. Além disso, o guanandi apresenta excelentes características silviculturais, uma vez que tem boa forma, com fuste reto e ausência de bifurcações e não apresenta problemas com pragas e doenças em nenhuma das fases de crescimento (PIOTTO et al., 2003).

Uma das etapas mais importantes da silvicultura é a definição da adubação ideal para cada espécie nas diversas etapas de seu desenvolvimento. Entretanto, Carvalho (2003), relata que a maioria das informações sobre espécies florestais nativas relaciona-se, principalmente, às características botânicas e dendrológicas e informações sobre exigências nutricionais são escassas.

nitrogênio é um dos nutrientes exigidos em grande quantidade pelas culturas agrícolas. Na maioria das espécies cultivadas, principalmente as de ciclo anual, o metabolismo do nitrogênio tem sido bastante estudado, porém para espécies florestais não ocorre o mesmo, sobretudo as espécies nativas (MARQUES et al., 2006).

Em razão da escassez de informações a respeito da demanda nutricional de espécies florestais nativas quanto à nutrição nitrogenada, neste trabalho, objetivouse avaliar o efeito de doses de nitrogênio no crescimento inicial e nutrição de plantas de guanandi (Calophyllum brasiliense Cambèss).

\section{MATERIAL E MÉTODOS}

O experimento foi conduzido em casa de vegetação, sem controle de temperatura e umidade relativa do ar, na área experimental do Departamento de Recursos Naturais/Ciência do Solo, da Faculdade de Ciências Agronômicas de Botucatu-SP/UNESP. O município está localizado na região centro-sul do Estado de São Paulo, nas coordenadas 22 52' 20', latitude S e 48'26’37' longitude W de Greenwich e altitude de $804 \mathrm{~m}$.
O tipo de solo utilizado foi classificado como Latossolo Vermelho distrófico (EMPRESA BRASILEIRA DE PESQUISA AGROPECUÁRIA - EMBRAPA, 2006). De acordo com o resultado da análise, o solo possuía as seguintes características: 289, 56 e $655 \mathrm{~g} \cdot \mathrm{dm}^{-3} \mathrm{de}$ argila, silte e areia, $\mathrm{pH}\left(\mathrm{CaCl}_{2}\right)=4, \mathrm{I}, \mathrm{M} . \mathrm{O}=19 \mathrm{~g} \cdot \mathrm{dm}^{-3}$, $\mathrm{P}$ (resina) $=2 \mathrm{mg} \cdot \mathrm{dm}^{-3} ; \quad \mathrm{Al}^{+3}=15 \mathrm{mmol} \cdot \mathrm{dm}^{-3}$, $\mathrm{H}+\mathrm{Al}=64 \mathrm{mmol}_{\mathrm{c}} \cdot \mathrm{dm}^{-3}, \mathrm{~K}^{+}=0,3 \mathrm{mmol} \cdot \mathrm{dm}^{-3}, \mathrm{Ca}^{2+}=$ $\mathrm{I} \mathrm{mmol} \cdot \mathrm{dm}^{-3}, \mathrm{Mg}^{2+}=0 \mathrm{mmol}_{\mathrm{c}} \cdot \mathrm{dm}^{-3}, \mathrm{SB}=\mathrm{I}, 3 \mathrm{mmol}_{\mathrm{c}}$. $\mathrm{dm}^{-3}$ e CTC $(\mathrm{T})=66 \mathrm{mmol} \cdot \mathrm{dm}^{-3}$, respectivamente; 0,$26 ; 1,0 ; 59 ; 0,3$ e $0,1 \mathrm{mg} \cdot \mathrm{dm}^{-3}$ de $B=0,26 \mathrm{mg} \cdot \mathrm{dm}^{-3}$, $\mathrm{Cu}=\mathrm{I}, 0 \mathrm{mg} \cdot \mathrm{dm}^{-3}, \mathrm{Fe}=59 \mathrm{mg} \cdot \mathrm{dm}^{-3}, \mathrm{Mn}=0,3 \mathrm{mg} \cdot \mathrm{dm}^{-3}$ e $Z n=0, I \mathrm{mg} \cdot \mathrm{dm}^{-3}$ e $\mathrm{V} \%=2 \%$.

A espécie utilizada foi o Guanandi, cujas mudas foram produzidas por sementes, as quais foram colocadas para germinar em caixa de areia e quando apresentaram dois pares de folhas, foram transplantadas para tubetes, preenchidos com substrato à base de casca de pinus. O tempo para a formação das mudas foi de seis meses, quando apresentavam em média $30 \mathrm{~cm}$ de altura e $6 \mathrm{~mm}$ de diâmetro.

O delineamento experimental utilizado foi inteiramente casualizado, com seis tratamentos e quatro repetições. Os tratamentos constituíram-se de cinco doses de N: 0, 40, 80, I 20 e $160 \mathrm{mg} \cdot \mathrm{dm}^{-3}$, mais o controle (sem adubação e calagem).

Com exceção do controle, todos os tratamentos receberam calagem para elevar a saturação por bases do solo de $60 \%$ e adubação com $150 \mathrm{mg} \cdot \mathrm{dm}^{-3}$ de $\mathrm{P}_{2} \mathrm{O}_{5}$, $80 \mathrm{mg} \cdot \mathrm{dm}^{-3}$ de $\mathrm{K}_{2} \mathrm{O}$. As fontes utilizadas de calagem, $\mathrm{N}$, $\mathrm{P}_{2} \mathrm{O}_{5}, \mathrm{~K}_{2} \mathrm{O}$ foram: calcário dolomítico, ureia, superfosfato simples, cloreto de potássio, respectivamente.

As mudas com 180 dias de idade foram plantadas em recipientes plásticos com capacidade de 50L, preenchidos com solo. Os vasos foram irrigados mantendo-se a capacidade de campo em torno de $70 \%$.

As características de crescimento foram avaliadas, mensalmente, por meio da medição de altura de plantas, desde o colo até o ápice das plantas, com o auxílio de régua graduada, e do diâmetro do caule, utilizando-se de um paquímetro digital.

Após 10 meses, todas as plantas foram separadas em folhas, caule e raízes. Foi avaliada a área foliar, utilizando-se todas as folhas de cada planta, a partir da imagem digitalizada em scanner pelo medidor Área Meter, marca Licor, modelo LI - 3600.

Realizou-se lavagem das partes das plantas com água destilada e, em seguida, as mesmas foram colocadas em estufa de circulação forçada de ar por 72 horas, na temperatura de $65^{\circ} \mathrm{C}$, até a obtenção de peso 
constante. A partir do peso seco das partes da planta, foi calculada a massa seca total.

Após a secagem e pesagem, as folhas e caules foram moídos e enviados para o Laboratório de Nutrição Mineral de Plantas da Faculdade de Ciências Agronômicas (FCA) da UNESP/Botucatu para a determinação dos teores de macro e micronutrientes, de acordo com metodologia proposta por Malavolta et al. (1997).

O acúmulo de nutrientes nas plantas foi calculado pelo produto entre a massa seca de folhas, caules e raízes pelos seus respectivos teores de nutrientes.

Os resultados obtidos foram submetidos à análise de variância e posterior análise de regressão, ajustando-se as equações aos dados obtidos, em função das doses de $\mathrm{N}$ e teste de média (Tukey), quando comparadas as doses de $\mathrm{N}$ com a planta controle. $\mathrm{O}$ programa estatístico utilizado foi o software SISVAR versão 4.2 (FERREIRA, 2003).

\section{RESULTADOS E DISCUSSÃO}

Houve influência das doses de $\mathrm{N}$ para altura e diâmetro de plantas após 10 meses de crescimento do guanandi, com ajuste quadrático e linear para altura e diâmetro do caule, respectivamente (Figura I).

A altura de plantas foi menor com o incremento nas doses de $\mathrm{N}$, a partir da dose de $33 \mathrm{mg} \cdot \mathrm{dm}^{-3}$ que proporcionou valor máximo de altura igual a $159 \mathrm{~cm}$ (Figura IA).

Com o aumento das doses, ocorreu redução do diâmetro do caule das plantas (Figura IB). Para cada 40 $\mathrm{mg} \cdot \mathrm{dm}^{-3}$ de nitrogênio adicionada ocorreu um redução no diâmetro do caule de 2,9 mm. De acordo com Resende et al. (1999), com o avanço do grupo sucessional, o estímulo ao crescimento proporcionado pela adubação é menos pronunciado e, algumas vezes, inexistente; tendência, em parte, atribuída ao crescimento mais lento, característico das espécies clímax. É também uma característica de adaptação a solos de baixa fertilidade.

Embora pouco expressivos, os modelos matemáticos de melhor ajuste para a massa seca de folhas e caule mostraram respostas positivas do guanandi em função das doses de $\mathrm{N}$ (Figura 2). As doses de $\mathrm{N}$ estimadas de 12,3 e $21,4 \mathrm{mg} \cdot \mathrm{dm}^{-3}$ de $\mathrm{N}$ proporcionaram os máximos valores, para $\mathrm{X}, \mathrm{Y}$ e $\mathrm{Z}$, respectivamente.

A maior massa seca foliar foi de 67,55 g.planta-1 na dose de $40 \mathrm{mg} \cdot \mathrm{dm}^{-3}$ de $\mathrm{N}$, enquanto que a menor foi de 13,88 g.planta-1 para a dose de $160 \mathrm{mg} \cdot \mathrm{dm}^{-3}$; a maior massa seca do caule foi de 67,22 g.planta-1 na dose de $40 \mathrm{mg} \cdot \mathrm{dm}^{-3}$ de $\mathrm{N}$, enquanto que a menor foi de $15,4 \mid \mathrm{g} \cdot$ planta $^{-1}$ para a
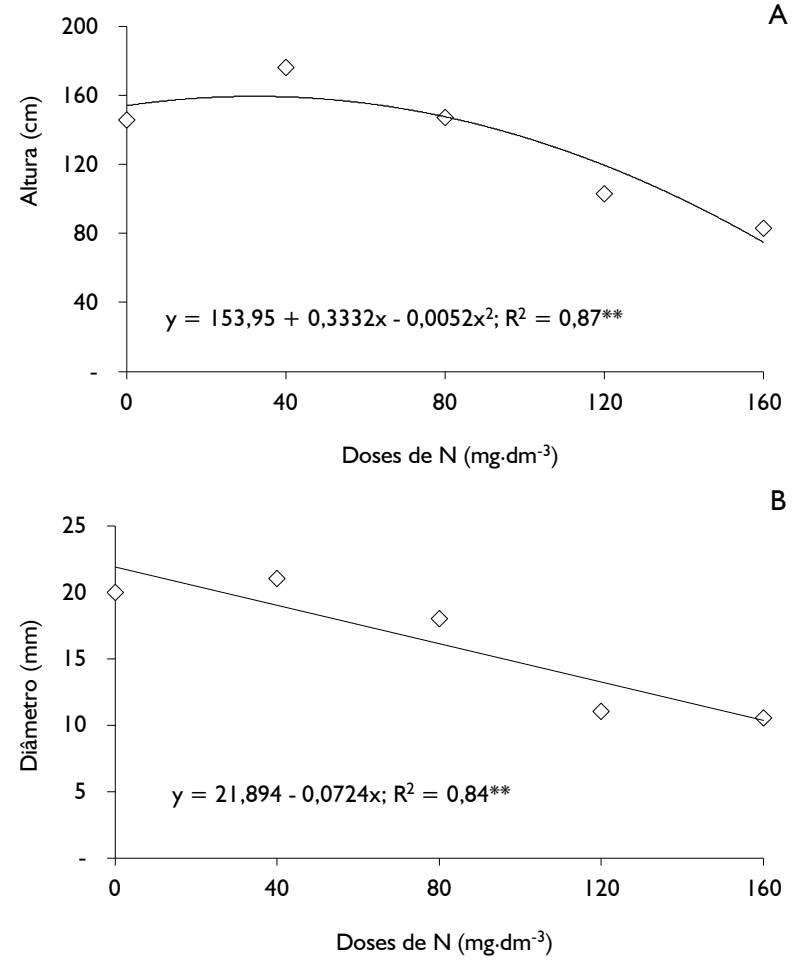

FIGURA I Altura das plantas (A) e diâmetro do caule (B) de guanandi com 10 meses de idade, em função de doses crescentes de N. Botucatu, FCA/UNESP, 2009.

FIGURE I Height (A) and diameter (B) of I0-month guanandi plants due to increasing $\mathrm{N}$ doses. Botucatu, FCA / UNESP, 2009.

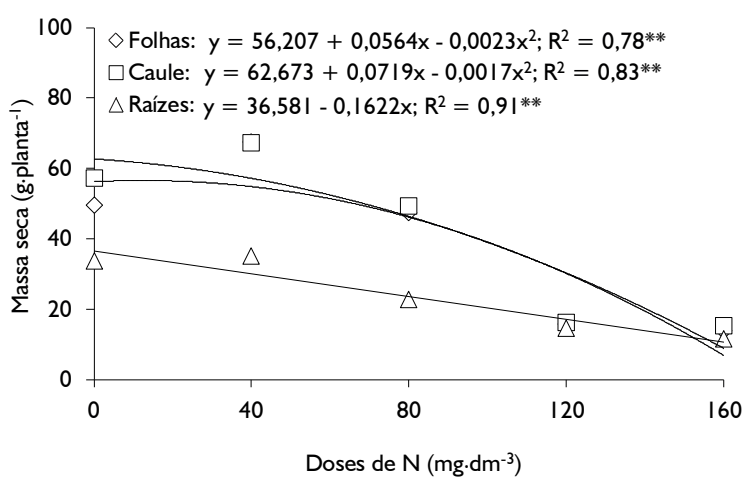

FIGURA 2 Massa seca das folhas $(\diamond)$, caule $(\square)$ e raízes $(\Delta)$ das plantas de guanandi com 10 meses de idade, em função de doses crescentes de N. Botucatu, FCA/ UNESP, 2009.

FIGURE 2 Leaves $(\diamond)$, stems $(\square)$ and roots $(\Delta)$ dry mass from I0-month guanandi plants due to increasing $N$ doses. Botucatu, FCA / UNESP, 2009.

dose de $160 \mathrm{mg} \cdot \mathrm{dm}^{-3}$; e a maior massa seca de raízes foi de $35,21 \mathrm{~g} \cdot$ planta $^{-1}$ na dose de $40 \mathrm{mg} \cdot \mathrm{dm}^{-3}$ de $\mathrm{N}$, enquanto que a menor foi de I I,64 g.planta-1 para a dose de $160 \mathrm{mg} \cdot \mathrm{dm}^{-3}$, apresentando o melhor crescimento na dose de $40 \mathrm{mg} \cdot \mathrm{dm}^{-3}$. 
De acordo com Marschner et al. (1996), o crescimento radicular pode ser favorecido em solos sob condições de deficiência de nutrientes. Gonçalves (1994) também verificou menor densidade de raízes de eucalipto em sítios menos férteis. $O$ incremento relativo no órgão de absorção pode ser uma estratégia para extrair o máximo de água e nutrientes presentes no solo. Vale salientar, também, que o tratamento que não recebeu $\mathrm{N}$ foi complementado com $\mathrm{P}$ e $\mathrm{K}$, sendo o fósforo considerado o nutriente mais importante no desenvolvimento de raízes.

As doses de $\mathrm{N}$ influenciaram, significativamente, a aérea foliar das plantas de guanandi. Os maiores valores de área foliar foram obtidos com a dose estimada de 46,4 $\mathrm{mg} \cdot \mathrm{dm}^{-3}$ de N (Figura 3).

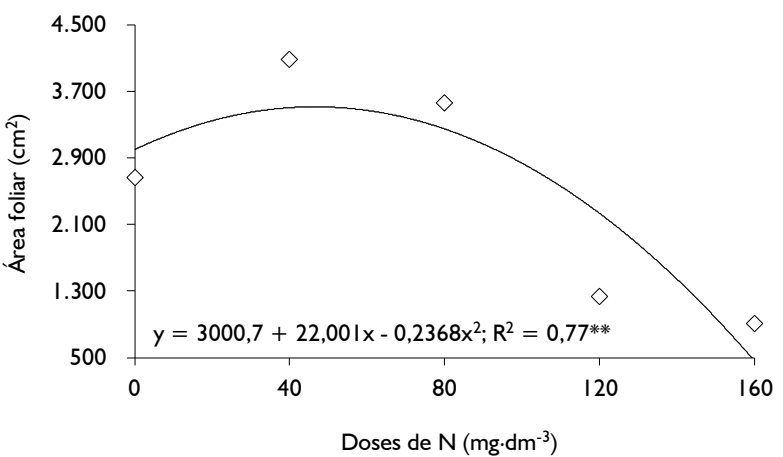

FIGURA 3 Área foliar de plantas de guanandi com 10 meses de idade, em função de doses crescentes de $\mathrm{N}$. Botucatu, FCA/UNESP, 2009.

FIGURE 3 Leaf area of 10-month guanandi plants due to increasing $\mathrm{N}$ doses. Botucatu, FCA / UNESP, 2009

Quando se compararam os tratamentos, verificou-se que o menor desenvolvimento da planta (altura, diâmetro, massa seca e área foliar) foi observado nas duas maiores doses de $\mathrm{N}\left(120\right.$ e $160 \mathrm{mg}^{-\mathrm{dm}^{-3}}$ ) e no tratamento controle que não recebeu nenhuma adubação (Tabela I). De forma semelhante em experimento com mudas de mogno brasileiro (Swietenia macrophylla), Tucci et al. (2009) observaram que a dose del $20 \mathrm{mg} \cdot \mathrm{dm}^{-3}$ de $\mathrm{N}$ afetou negativamente todas as características de crescimento avaliadas, assim como Favare (2010) observou redução no crescimento de plantas de teca (Tectona grandis) nas doses de 120 e $160 \mathrm{mg} \cdot \mathrm{dm}^{-3}$.

As plantas, de modo geral, respondem bem à adubação nitrogenada, sendo que o efeito externo do nitrogênio mais visível é a vegetação verde e abundante. Porém, o excesso de $\mathrm{N}$ é prejudicial; sendo assim, a dose fornecida à cultura deve ser bem equilibrada em relação à quantidade de outros nutrientes. A espécie estudada demonstrou baixa necessidade de nitrogênio, nesta etapa do seu crescimento, sendo que a menor dose fornecida $\left(40 \mathrm{mg} \cdot \mathrm{dm}^{-3}\right)$ foi suficiente para um bom crescimento da planta.

TABELA I Altura, diâmetro, massa seca de folhas caule e raízes e área foliar de plantas de guanandi com 10 meses de idade, em função dos tratamentos. Botucatu, FCA/UNESP, 2009.

TABLE I Height; diameter; stem, leaves and roots dry mass and leaf area of 10-month guanandi plants due to increasing $\mathrm{N}$ doses, due to the applications. Botucatu, FCA / UNESP, 2009

\begin{tabular}{|c|c|c|c|c|c|c|}
\hline \multirow{3}{*}{ T } & \multirow{3}{*}{$\begin{array}{c}\text { Altura } \\
----c m---\end{array}$} & \multirow{3}{*}{$\begin{array}{l}\text { Diâmetro } \\
\text {---mm--- }\end{array}$} & \multicolumn{3}{|c|}{ Massa seca } & \multirow{2}{*}{ Área foliar } \\
\hline & & & Folhas & Caules & Raízes & \\
\hline & & & & -g.planta & & \\
\hline 0 & $145,50 \mathrm{ab}$ & & $49,62 a$ & & $33,82 a$ & \\
\hline 40 & & & $67,55 a$ & & 35,2 & \\
\hline 80 & $147,17 \mathrm{ab}$ & 17,6 & $47,42 a$ & 49,3 & $22,67 b$ &, $99 a$ \\
\hline 120 & $102,33 \mathrm{bc}$ & II, I & $15,68 b$ & $16,23 b$ & I4,70bc & $1230,76 \mathrm{bc}$ \\
\hline 160 & & & $13,88 b$ & $15,4 \mathrm{Ib}$ & $11,64 c$ & $906,80 c$ \\
\hline $\mathrm{Te}$ & & & $23,0 \mathrm{lb}$ & $2 I, 84 b$ & $18,28 b c$ & \\
\hline CV\% & & & 28,04 & 22,96 & 16,90 & \\
\hline
\end{tabular}

Letras iguais na linha não diferem entre si pelo teste de Tukey a $5 \%$ de probabilidade. T - tratamento; Te - testemunha.

As concentrações de $\mathrm{N}$ nas folhas e caule foram influenciadas positivamente pelas doses crescentes de $\mathrm{N}$, apresentando comportamento linear crescente em função das doses aplicadas (Figura 4).

Para o $\mathrm{K}$ e $\mathrm{S}$, nas folhas, também se verificou aumento na concentração conforme se aumentaram as doses de $\mathrm{N}$, demonstrando um efeito de concentração de nutrientes nas folhas, efeito esse amplamente relatado na literatura (JARREL; BEVERLY, 198I). Para a concentração de $\mathrm{K}$ no caule, o ajuste foi quadrático, quando o máximo valor foi obtido com a dose estimada de $88 \mathrm{mg} \cdot \mathrm{dm}^{-3}$ de N.

Não houve diferença significativa para a concentração de $\mathrm{Ca}$ e $\mathrm{Mg}$ na parte aérea das plantas. Para as concentrações desses nutrientes no caule, verificaram-se valores maiores na dose zero, pelo maior desenvolvimento das plantas, e nas maiores doses de $\mathrm{N}$, pelo efeito de concentração, já que houve efeito negativo das doses elevadas de nitrogênio no desenvolvimento das plantas.

Para os micronutrientes, houve influência das doses de $\mathrm{N}$ apenas para as concentrações de $\mathrm{Cu}$ nas folhas e de Mn nas folhas e caule das plantas de guanandi (Figura 5).

Assim como ocorreu para as concentrações de $\mathrm{Ca}$ e $\mathrm{Mg}$, verificaram-se concentrações maiores de Cu e $\mathrm{Zn}$ na dose zero e nas maiores doses de $\mathrm{N}$. 

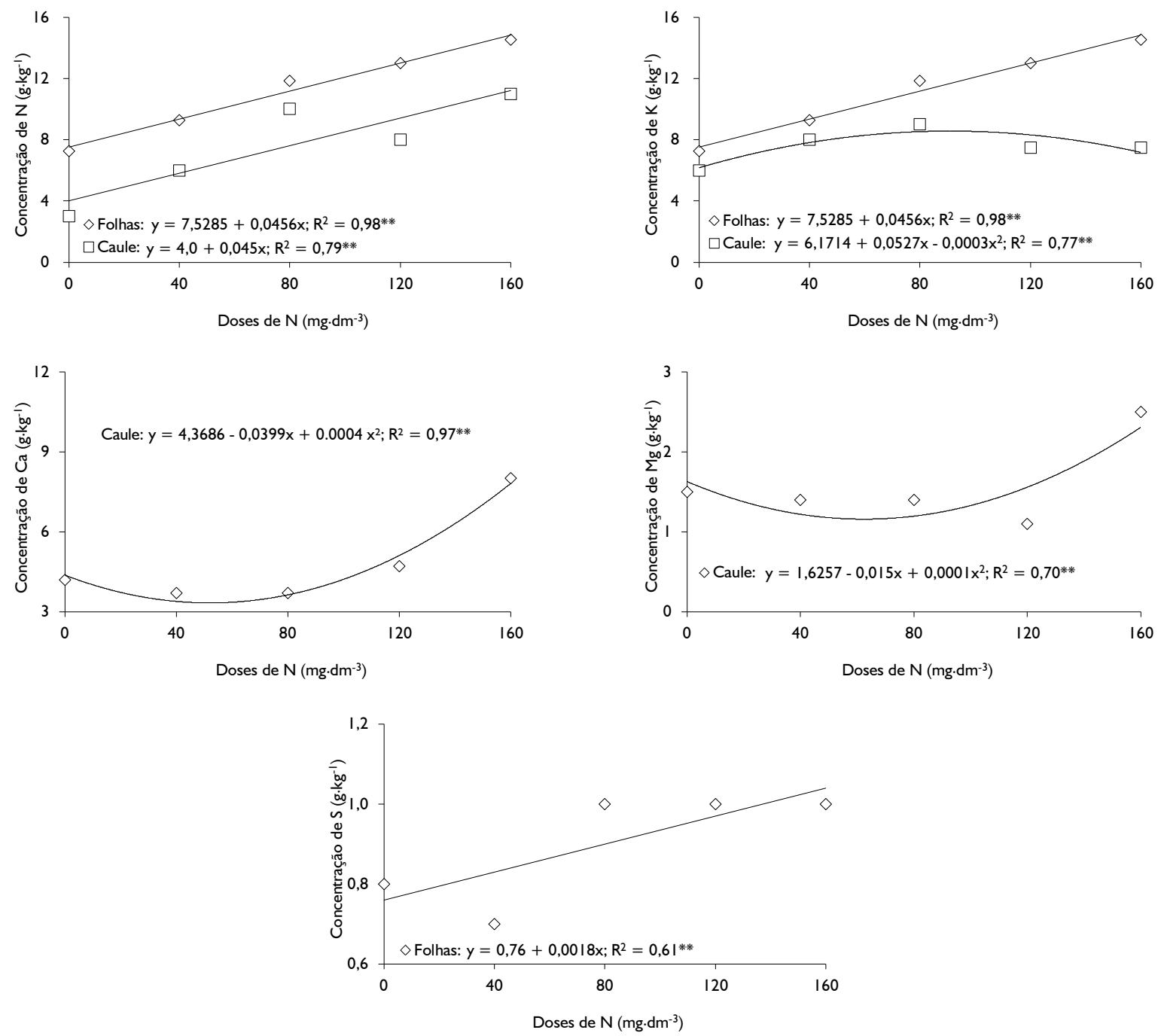

FIGURA 4 Teores de macronutrientes nas folhas $(\diamond)$ e caule $(\square)$ de guanandi aos 10 meses de idade, em função de doses crescentes de N. Botucatu, FCA/UNESP, 2009.

FIGURE 4 Macronutrients levels on leaves $(\diamond)$ and stems $(\square)$ of I0-month guanandi plants due to increasing $\mathrm{N}$ doses. Botucatu, FCA / UNESP, 2009.
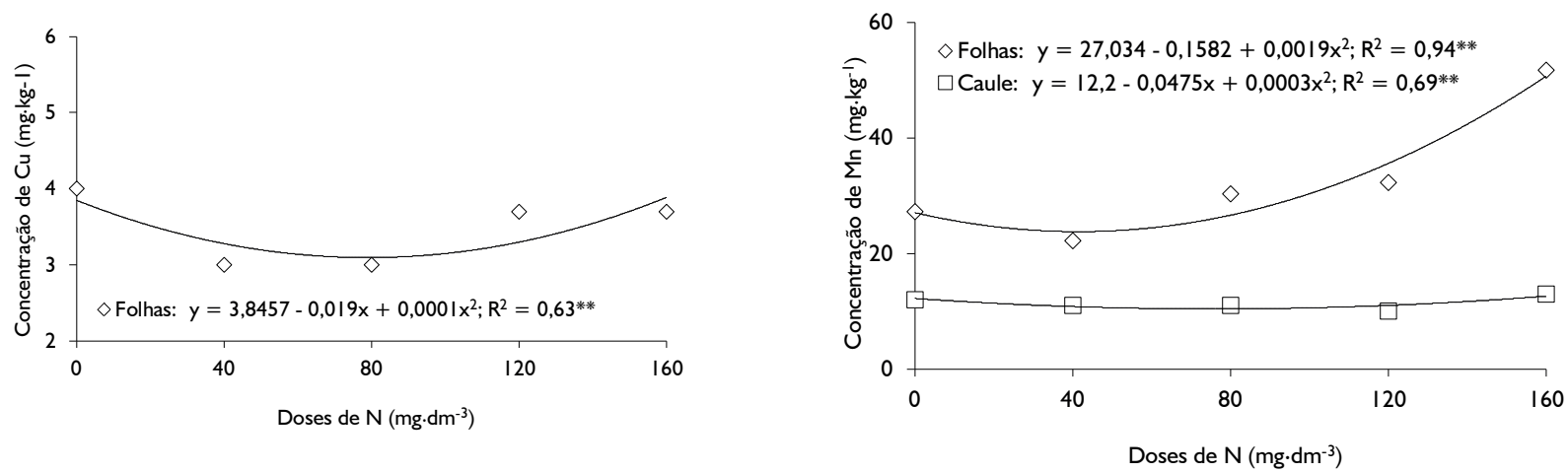

FIGURA 5 Teores de micronutrientes nas folhas $(\diamond)$ e caule( $(\square)$ de guanandi aos 10 meses de idade, em função de doses crescentes de N. Botucatu, FCA/UNESP, 2009.

FIGURE 5 Micronutrient levels on leaves $(\diamond)$ and stems $(\square)$ of I0-month guanandi plants due to increasing $N$ doses. Botucatu, FCA/ UNESP, 2009. 
Comaaplicação de doses crescentes de $\mathrm{N}$, houve resposta em termos de acúmulo de macronutrientes nas folhas e caules de plantas de guanandi. Para o acúmulo nas folhas, verifica-se comportamento quadrático (Figura 6).

Os maiores valores de acúmulo de N, P, K, Ca, $\mathrm{Mg}$ e $\mathrm{S}$ nas folhas foram obtidos com as doses estimadas de $46,50,40,20,12$ e $3 \mathrm{mg} \cdot \mathrm{dm}^{-3}$, respectivamente.

Tucci et al. (2009) também verificaram que o $\mathrm{N}$ favoreceu a absorção e/ou utilização do $\mathrm{P}$ e do Ca por plantas de mogno submetidas a doses crescentes de $\mathrm{N}$. Os autores observaram correlação positiva e significativa entre doses de $\mathrm{N}$ aplicado $\times \mathrm{P}$ acumulado e doses de $\mathrm{N}$ x $\mathrm{Ca}$ acumuladas na massa seca das folhas.

As respostas em acúmulo de N, P, Ke S no caule de plantas de guanandi apresentaram melhor ajuste ao modelo quadrático. As doses para os máximos acúmulos foram estimadas em 70, 50, 53 e $8 \mathrm{mg} \cdot \mathrm{dm}^{-3}$, respectivamente. Para $\mathrm{Ca}$ e $\mathrm{Mg}$ o efeito foi linear decrescente.

As plantas responderam à aplicação de $\mathrm{N}$ com o incremento inicial de acúmulo dos nutrientes estudados e, a partir da dose de $80 \mathrm{mg} \cdot \mathrm{dm}^{-3}$ a resposta à adubação passou a diminuir. A diminuição do acúmulo desses nutrientes na parte aérea das mudas deve-se ao fato de que, em doses
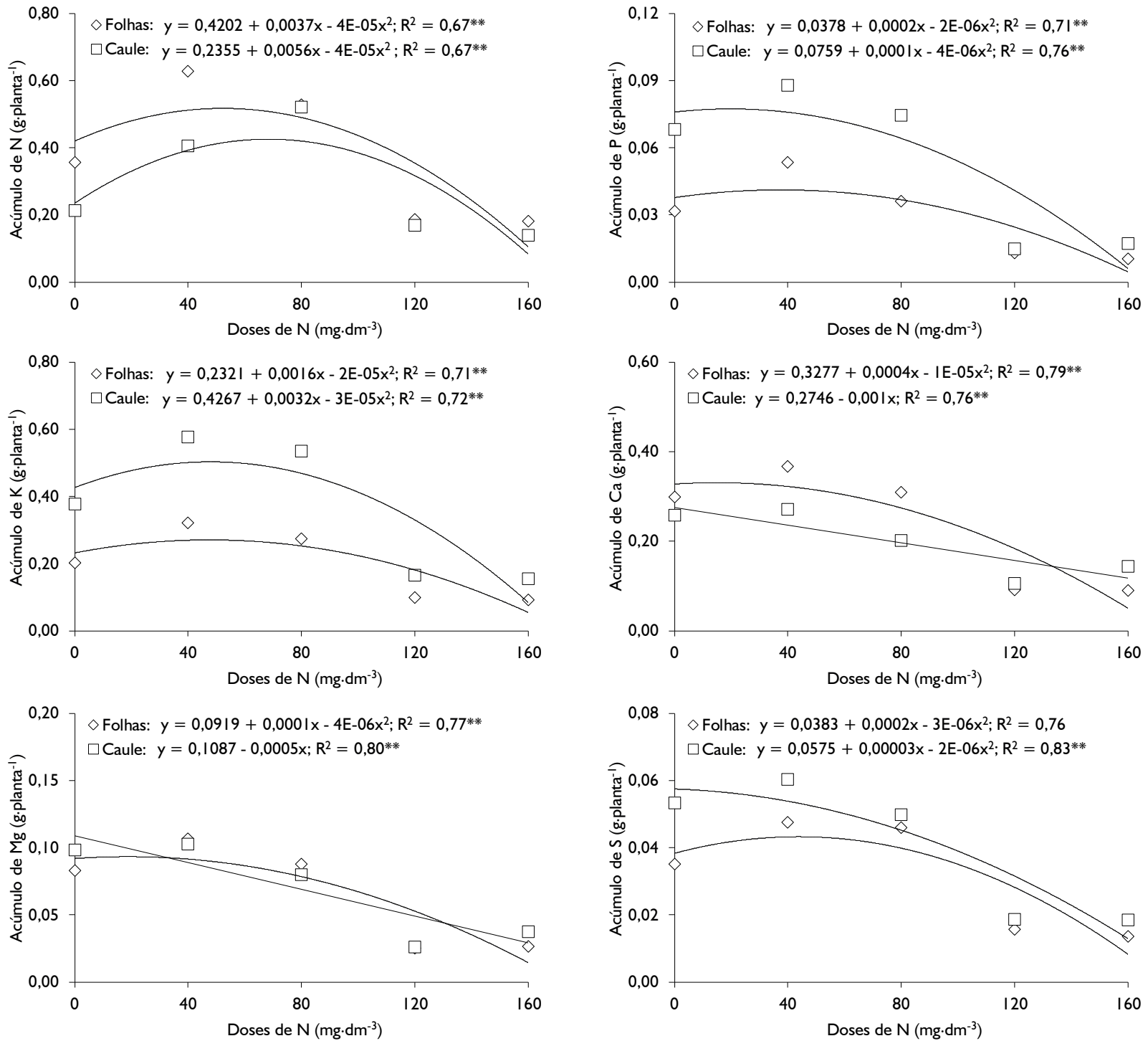

FIGURA 6 Acúmulo de macronutrientes nas folhas $(\diamond)$ e caule $(\square)$ de plantas de guanandi aos 10 meses de idade, em função de doses crescentes de N. Botucatu, FCA/UNESP, 2009.

Accumulation of nutrients on leaves $(\diamond)$ and stems $(\square)$ of 10 -month guanandi plants due to increasing $N$ doses.

FIGURE 6 Botucatu, FCA / UNESP, 2009. 
maiores de N, o crescimento das plantas foi comprometido, como verificado nas avaliações realizadas de altura, massa seca e área foliar.

As doses crescentes de $\mathrm{N}$ influenciaram, significativamente, no acúmulo de micronutrientes nas folhas e caules de plantas de guanandi (Figura 7). Houve ajuste quadrático apenas para os acúmulos de Fe e Mn nas folhas, onde as doses estimadas de 16,7 e 16,2 $\mathrm{mg} \cdot \mathrm{dm}^{-3}$ proporcionaram os máximos valores, respectivamente. Para o acúmulo dos demais micronutrientes nas folhas e caules, verifica-se uma redução dos valores com o aumento das doses de $\mathrm{N}$.
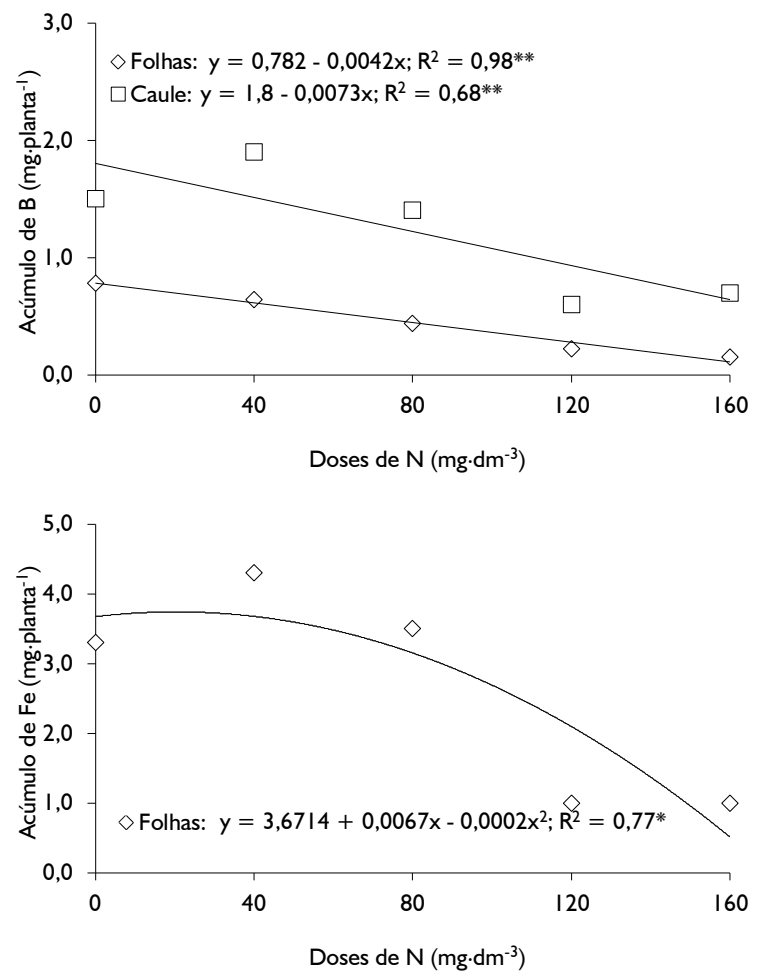

\section{CONCLUSÃO}

Recomenda-se a dose de $40 \mathrm{mg} \cdot \mathrm{dm}^{-3}$ ou 80 $\mathrm{kg} \cdot \mathrm{ha}^{-1}$ de $\mathrm{N}$ na época de plantio, para suprir a necessidade de nitrogênio no crescimento inicial do guanandi.

\section{AGRADECIMENTOS}

Agradeço à Empresa Tropical Flora Reflorestadora, situada em Garça - SP, pela doação das mudas de guanandi utilizadas neste experimento.
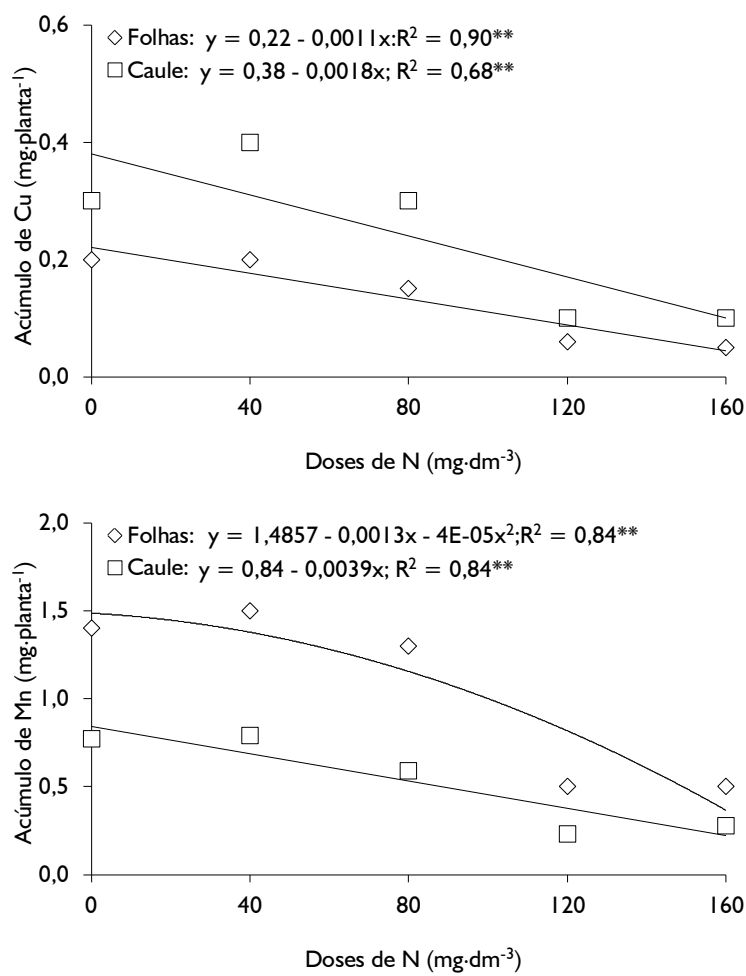

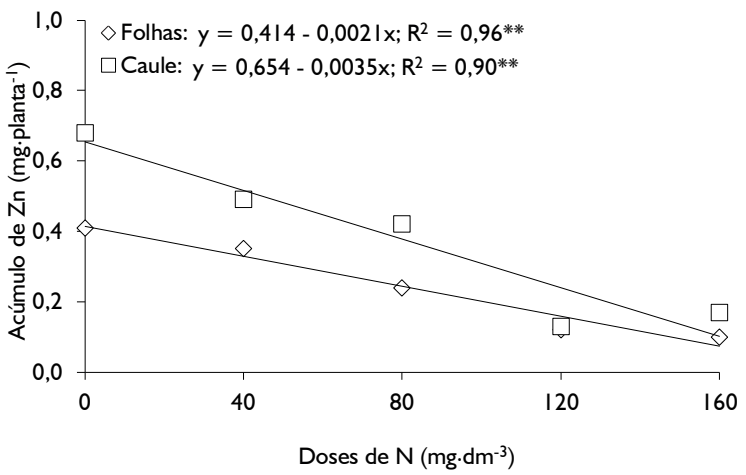

FIGURA 7 Acúmulos de micronutrientes nas folhas de guanandi aos 10 meses de idade, em função de doses crescentes de $\mathrm{N}$. Botucatu, FCA/UNESP, 2009.

Micronutrient accumulation rates on leaves of 10-month guanandi plants due to increasing $\mathrm{N}$ doses. Botucatu, FCA / FIGURE 7 UNESP, 2009. 


\section{REFERÊNCIAS}

CARVALHO, P. E. R. Espécies arbóreas brasileiras. Brasília: EMBRAPA Informação Tecnológica; Colombo: EMBRAPA Florestas, 2003.

EMPRESA BRASILEIRA DE PESQUISA AGROPECUÁRIA. Centro Nacional de Pesquisa de Solos. Sistema brasileiro de classificação de solos. 2. ed. Rio de Janeiro, 2006. 306 p.

FAVARE, L. G. Doses crescentes de nitrogênio, fósforo, potássio e diferentes níveis de saturação por bases em relação ao desenvolvimento e nutrição mineral de teca (Tectona grandis L.F.) sob condições de vaso. 2010. 106 f. Dissertação (Mestrado em Ciência Florestal) Universidade Estadual Paulista, Botucatu, 2010.

FERREIRA, D. F. Sisvar. Versão 4.2. Lavras: DEX/UFLA, 2003.

GONÇALVES, J. L. M. Características do sistema radicular de absorção do Eucalyptus grandis sob diferentes condições edáficas. 1994. 84 p. Tese (Livre Docência em Ciências Agrárias) - Escola Superior de Agricultura "Luis de Queiroz", Piracicaba, 1994.

JARRELL, W. M.; BEVERLY, R. B. The dilution effect in plant nutrition studies. Advance Agronomy, New York, v. 34, p. 197-224, 1981.

MALAVOLTA, E.; VITTI, C.; OLIVEIRA, S. A. Avaliação do estado nutricional das plantas: princípios e aplicações. Piracicaba: POTAFÓS, 1997. 319 p.
MARQUES, V. B.; PAIVA, H. N.; GOMES, J. M.; NEVES, J. C. L.; BERNARDINO, D. C. S. Efeito de fontes e doses de nitrogênio sobre o crescimento inicial e qualidade de mudas de jacarandá-da-bahia (Dalbergia nigra (Vell.) Fr. All. ex Benth.). Revista Árvore, Viçosa, v. 30, n. 5, p. 725-735, set./out. 2006.

MARSCHNER, H.; KIRKBY, E. A.; CAKMAK, I. Effect of mineral nutritional status on shoot-root partioniting of photoassimilates and cycling of mineral nutrients. Journal of Experimental Botany, Oxford, v. 47, p. 1255-1263, 1996. Special issue.

PIOTTO, D.; MONTAGNINI, F.; UGALDE, L.; KANNINEN, $M$. Growth and effects of thinning of mixed and pure plantations with native trees in humid tropical Costa Rica. Forest Ecology and Management, Amsterdam, v. I77, p. 427-439, 2003.

RESENDE, A. V.; FURTINI NETO, A. E.; MUNIZ, J. A.; CURI, N.; FAQUIN, V. Crescimento inicial de espécies florestais de diferentes grupos sucessionais em resposta a doses de fósforo. Pesquisa Agropecuária Brasileira, Brasília, v. 34, n. I I, p. 207I-208I, nov. 1999.

TUCCI, C. A. F.; LIMA, H. N.; LESSA, J. F. Adubação nitrogenada na produção de mudas de mogno (Swietenia macrophylla King). Acta Amazônica, Manaus, v. 39, n. 2, p. 289-294, 2009. 